\title{
Reduction of Waveform Distortions in Bispectrum-Based Signal Reconstruction System
}

\author{
A.V. Totsky ${ }^{1}$, P.Yu. Kostenko ${ }^{2}$, J.T. Astola ${ }^{3}$, K.O. Egiazarian ${ }^{3}$, \\ and I.V. Kurbatov ${ }^{1}$ \\ ${ }^{1}$ National Aerospace University, Chkalova Str. 17, 61070, Kharkov, Ukraine, \\ ${ }^{2}$ Kharkov Air Force University, Klochkovskaya Str. 228, 61165, Kharkov, Ukraine, \\ ${ }^{3}$ Signal Processing Laboratory, P.O. Box 553, FIN-33101, Tampere, Finland,
}

\begin{abstract}
The problem of reconstruction an unknown signal waveform from bispectrum is considered. A new approach based on utilization of continuous sine and cosine functions instead of conventional discontinuous bispectrum phase function in signal reconstruction recursive algorithm is proposed. The performance of the developed signal waveform reconstruction algorithm is analyzed by numerical simulation for realvalued deterministic discrete-time signals containing discontinuities of the first kind in the cases of different sampling rates. Simulation studies demonstrate that significant reconstructed signal quality enhancement occurs with the proposed simple and computationally efficient algorithm.
\end{abstract}

\section{INTRODUCTION}

Several algorithms that directly or indirectly use phase information have been proposed for reconstruction a signal from its bispectrum [1-6]. The authors of the cited papers assume that it is necessary to compute the phase bispectrum for signal reconstruction directly [1-3]; or to restore a signal without direct consideration of phase bispectrum but using phase information inherent in signal log-bispectrum (bicepstrum) [4] or differential cepstrum [5]; or to recover the phase of a system transfer function from any pair of consecutive phase bispectrum slices [6]. Note that, on one hand, errorless recovery of phase information is possible only under certain conditions and constraints imposed on the processed signals and, on the other hand, the errors that arise in phase recovery may provoke significant distortions in reconstructed signals. For example, 2-D (see references [1-3]) or 1-D (see nonparametric algorithm described in [4] and reference [6]) phase unwrapping is required for reconstruction the true phase of a signal Fourier spectrum. Effectiveness of the parametric algorithm [4] largely depends on the location of signal zeros and poles towards the unit circle on zplane. It does not allow zeros or poles on the unit circle since the parametric algorithm [4] is based on the cepstral coefficients that are not defined in this case. The additional a priori information in the form of signal power cepstrum, as well as a priori knowledge of signal magnitude to transform the processed sequence to minimum/maximum phase signal are necessary for successful operation of the parametric algorithm [4]. The bispectrum iterative reconstruction algorithm [4] also 
operates under certain conditions, such as a phase of bispectrum is supposed to be known and, hence, differences of cepstrum coefficients are also known. However, high convergence rate in iterative algorithm [4] is possible when a priori information about the signal is available. The strict requirements must be imposed on differentiability of z-transform of processed sequence that cannot have zeros on the unit circle in algorithm [5] using differential cepstrum.

In our opinion, though phase information can be interesting for a number of applications, its direct measurement is unnecessary for solving bispectrum-based signal reconstruction problem. In this paper we develop a new approach to solving this problem without addressing to direct phase calculations. Instead of traditional approaches and their above-mentioned intrinsic limitations, we propose to use recursive signal reconstruction procedures for quadrature components of the complexvalued normalized bispectrum and signal Fourier spectrum.

\section{ANALYSIS OF TYPICAL DISTORTIONS OF SIGNAL RECONSTRUCTED FROM BISPECTRUM}

In this paper we are focusing on methodical deterministic errors that are typical for bispectrum-based signal reconstruction techniques using direct bispectrum and Fourier phase calculations. Therefore, we consider signal reconstruction in deterministic problem statement.

According to the direct method [7], for a real-valued, deterministic, discrete-time and time-limited signal $x_{i}(i=0,1, \ldots, I-1$, and, traditionally, unitary and uniform temporal sampling is assumed) its bispectrum $B_{p, q}$ can be defined as the following 2D complex-valued function

$$
B_{p, q}=X_{p} X_{q} X_{p+q}^{*}=B_{p, q}^{\mathrm{Re}}+j B_{p, q}^{\operatorname{Im}}=\left|B_{p, q}\right| e^{j \beta} p, q
$$

where $X_{p}=\left|X_{p}\right| e^{j \varphi} p$ is the discrete Fourier transform of $x_{i} ; B_{p, q}^{\mathrm{Re}}$ and $B_{p, q}^{\mathrm{Im}}$ are the real and imaginary parts, respectively; $\left|B_{p, q}\right|=\sqrt{\left(B_{p, q}^{\mathrm{Re}}\right)^{2}+\left(B_{p, q}^{\mathrm{Im}}\right)^{2}}$ is the magnitude bispectrum; $\beta_{p, q}=\arctan \frac{B_{p, q}^{\mathrm{Im}}}{B_{p, q}^{\mathrm{Re}}}$ is the phase bispectrum determined within the main interval of inverse tangent function, i.e., $\beta_{p, q} \in(-\pi, \pi] ; p=0,1, \ldots, I-1$ and $q=0$, $1, \ldots, I-1$ are the independent frequency indices; $\varphi_{p}$ and $\left|X_{p}\right|$ are the signal phase and magnitude Fourier spectra, respectively; * denotes complex conjugation; $j=\sqrt{-1}$. 
To recover the magnitude $\left|\hat{X}_{p}\right|$ and phase $\hat{\varphi}_{p}$ of the Fourier spectrum of the signal from its measured bispectrum (1), it is common [1-4] to use recursive solution of the following equations

$$
\begin{aligned}
& \left|\hat{X}_{p+q}\right|=\frac{\left|B_{p, q}\right|}{\left|\hat{X}_{p}\right|\left|\hat{X}_{q}\right|}, \\
& \hat{\varphi}_{p+q}=\hat{\varphi}_{p}+\hat{\varphi}_{q}-\arctan \frac{B_{p, q}^{\mathrm{Im}}}{B_{p, q}^{\mathrm{Re}}} .
\end{aligned}
$$

Since the procedure (2) usually permits to recover a magnitude Fourier spectrum without any distortions, we are focusing on the typical phase spectrum recovery procedure (3) that will be compared below to the proposed algorithm. First, let us pay attention to the following important peculiarities of the conventional bispectrum-based signal reconstruction technique.

1) Due to the well-known bispectrum translation invariance property, the linear Fourier phase term $\hat{\varphi}_{1}$ cannot be calculated from (3) and it is commonly supposed to be $\hat{\varphi}_{1}=0$. Hence, reconstructed signal $\hat{x}_{i}$ is always centered relatively to its center of gravity.

2) Hermitian symmetry of the phase Fourier spectrum of real-valued signal defined as $\hat{\varphi}_{0}=0$ and $\hat{\varphi}_{p}=-\hat{\varphi}_{-p}$ allows eliminating from the recovery procedure (3) the phase bispectrum samples that lie on the axis $p=0$ because they are always zero, i.e., $\beta_{0, q}=\varphi_{0}+\varphi_{q}-\varphi_{q}=\varphi_{0}=0$.

3) Recursive strategy proposed in [1] and repeated later by many authors for signal Fourier phase spectrum recovery by procedure (3) is implemented practically for bispectrum samples that belong to the main bispectrum triangular domain that is limited by the inequalities as $1 \leq p \leq q<N, p+q \leq N$ for odd $N=(I / 2)-1$ ( $I$ is supposed to be even). Note that two different possible sequences of recursive steps are possible. The first one is parallel to the frequency axis $p=0$ and the second one is parallel to the axis $q=0$. These two different data processing ways are represented by the following corresponding two equations 


$$
\begin{aligned}
& \hat{\varphi}_{2}=-\beta_{1,1} \\
& \hat{\varphi}_{3}=\hat{\varphi}_{2}-\beta_{1,2} \\
& \hat{\varphi}_{4}=\hat{\varphi}_{3}-\beta_{1,3} \\
& \hat{\varphi}_{5}=\hat{\varphi}_{4}-\beta_{1,4} \\
& \hat{\varphi}_{N}=\hat{\varphi}_{N-1}-\beta_{1, N-1} \\
& \hat{\varphi}_{4}=2 \hat{\varphi}_{2}-\beta_{2,2} \\
& \hat{\varphi}_{5}=\hat{\varphi}_{2}+\hat{\varphi}_{3}-\beta_{2,3} \\
& \hat{\varphi}_{N}=\hat{\varphi}_{2}+\hat{\varphi}_{N-2}-\beta_{2, N-2} \\
& \hat{\varphi}_{2}=-\beta_{1,1} \\
& \hat{\varphi}_{3}=\hat{\varphi}_{2}-\beta_{1,2} \\
& \hat{\varphi}_{4}=2 \hat{\varphi}_{2}-\beta_{2,2} \\
& \hat{\varphi}_{4}=\hat{\varphi}_{3}-\beta_{1,3} \\
& \hat{\varphi}_{N}=\hat{\varphi}_{\frac{N-1}{2}}+\hat{\varphi}_{\frac{N+1}{2}}-\beta_{\frac{N-1}{2}, \frac{N+1}{2}} \\
& \hat{\varphi}_{5}=\hat{\varphi}_{4}-\beta_{1,4} \\
& \hat{\varphi}_{N}=\hat{\varphi}_{N-1}-\beta_{1, N-1}
\end{aligned}
$$

Analysis of the procedures (4a) (the way parallel to the axis $p=0$ ) and (4b) (the way parallel to the axis $q=0$ ) shows that the samples of $\hat{\varphi}_{p+q}$ for $p+q \geq 4$ that have the same indices (correspond to the same frequencies) can be calculated independently several times and, hence, can be defined in several different ways. This well-known redundancy peculiarity is employed in several algorithms (see, for example [1,2,6]) for noise suppression by averaging the corresponding Fourier phase samples in noise environment. It was shown in [1], that the procedures (4a) and (4b) may produce different results at the reconstruction system output in case of noise presence.

4) The behavior of the function $\beta_{p, q}$ is characterized by discontinuities (wrappings) in $-\pi$ and $\pi$ rad that may cause the abruptions in the recovered signal phase Fourier spectrum $\hat{\varphi}_{p+q}$. It should be especially noted, that the behavior (steepness and oscillation frequency) of the functions $B_{p, q}^{\mathrm{Re}}$ and $B_{p, q}^{\mathrm{Im}}$ along the frequency axes $p=0$ and $q=0$ may vary considerably depending on a processed signal waveform. For fixed uniform sampling interval $\Delta p=\Delta q=1 / I$ in the bispectrum plane $(p, q)$, sampling errors of the function $\beta_{p, q}$ are dependent on its behavior that is usually unknown in practice. Thus, for fixed sampling interval that is usually limited by computer processing rate and memory capacity and under a priori unknown Nyquist frequency, one should expect the distortions of Fourier phase spectrum recovered by procedures (4a) or (4b).

Let us investigate the accuracy of reconstructed signal that is achieved by the conventional algorithms utilizing direct phase measurements. As typical example, we consider the algorithm developed in paper [1]. For this purpose, we introduce the following quantitative measure of distortion for the reconstructed waveform 


$$
\delta=\min _{t}\left\{\frac{\sum_{i=0}^{I-1}\left|x_{i-t}-\hat{x}_{i}\right|}{\sum_{i=0}^{I-1}\left|x_{i}\right|}\right\}
$$

where minimization over $t=0,1, \ldots, I-1$ is used to take into account possible temporal shift in the reconstructed signal $\hat{x}_{i}$.

We now study the methodical distortions that are typical for conventional algorithm [1] and its implementation in a number of papers.

First, we consider the problem of reconstruction of the single rectangular pulse signals with different pulse lengths $\Delta t$ (in practice, signal waveform and $\Delta t$ are $a$ priori unknown) observed in the fixed limited sample grid, for example, of $I=256$ samples. Let us study the signal centered with respect to the point of origin. In this case, distortion values calculated according to (5) for procedures (4a) and (4b) are represented in Table I.

It should be stressed, tat procedures (4a) and (4b) cause the same distortions and analysis of the distortion values given in Table I demonstrates that $\delta$ tends to increase with $\Delta t$ increasing.

As a typical example, the signal of the length of $\Delta t=37$ samples and the amplitude of $A=1$ reconstructed by the procedure (4a) or (4b) is shown in Fig. 1. It is clearly seen from Fig. 1, that original rectangular signal waveform is sufficiently distorted.

Table 1: Waveform distortions $\delta$ calculated for the procedures (4a) and (4b)

\begin{tabular}{|c|c|c|c|c|c|c|c|c|c|c|c|}
\hline$\Delta t$ & 9 & 13 & 17 & 21 & 25 & 29 & 33 & 37 & 41 & 45 & 49 \\
\hline$\delta$ & $\begin{array}{c}0.00 \\
02\end{array}$ & $\begin{array}{c}0.00 \\
3\end{array}$ & $\begin{array}{c}0.01 \\
7\end{array}$ & $\begin{array}{c}0.05 \\
9\end{array}$ & $\begin{array}{c}0.10 \\
5\end{array}$ & $\begin{array}{c}0.14 \\
5\end{array}$ & $\begin{array}{c}0.09 \\
7\end{array}$ & $\begin{array}{c}0.17 \\
3\end{array}$ & $\begin{array}{c}0.15 \\
6\end{array}$ & $\begin{array}{c}0.20 \\
4\end{array}$ & $\begin{array}{c}0.19 \\
2\end{array}$ \\
\hline
\end{tabular}

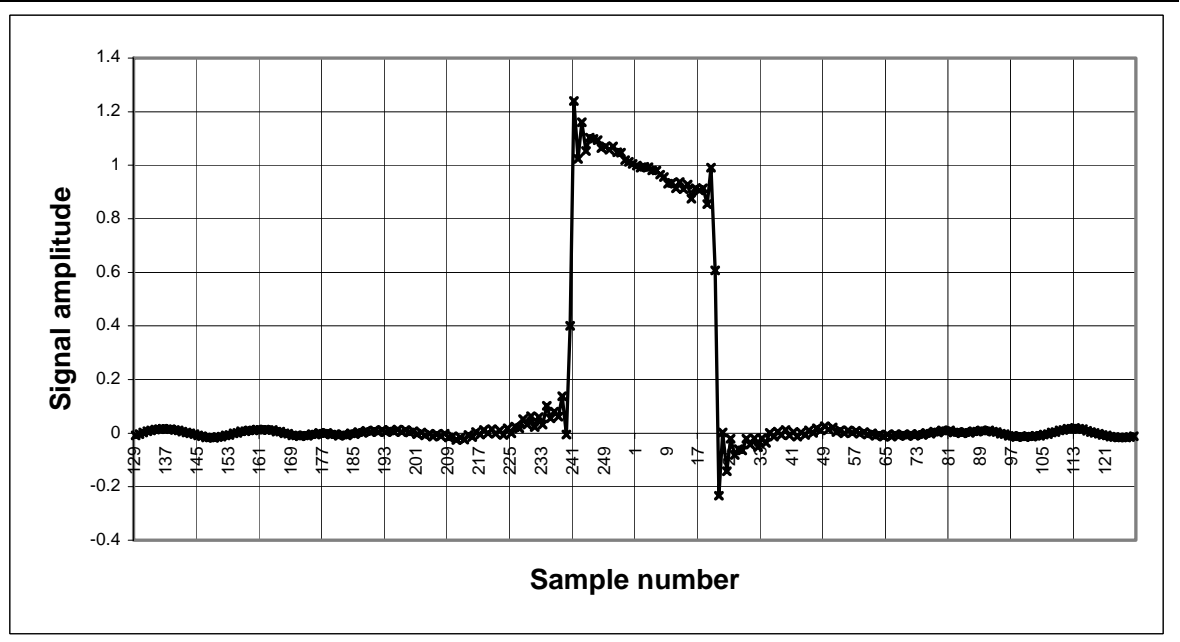

FIGURE 1. Signal reconstructed by the procedure (4a) or (4b), $\delta=0.173$. 
Let us consider now the second type of the test signal given in the form of two short rectangular pulses. Suppose that pulses have the same length of $\Delta t_{1}=\Delta t_{2}=3$ samples, different amplitudes equal to $A_{1}=1$ and $A_{2}=4$ and mutual pulse center shift equal to 7 samples.

The two-pulse signal reconstructed by the procedure (4a) or (4b) is shown in Fig. 2. As it is seen from Fig. 2, the reconstructed signal waveform is sufficiently distorted. Note that the distortions (5) calculated for this two-pulse test signal reconstructed by the procedure (4b) and (4b) are equal to approximately the same value $\delta \approx 0.6515$.

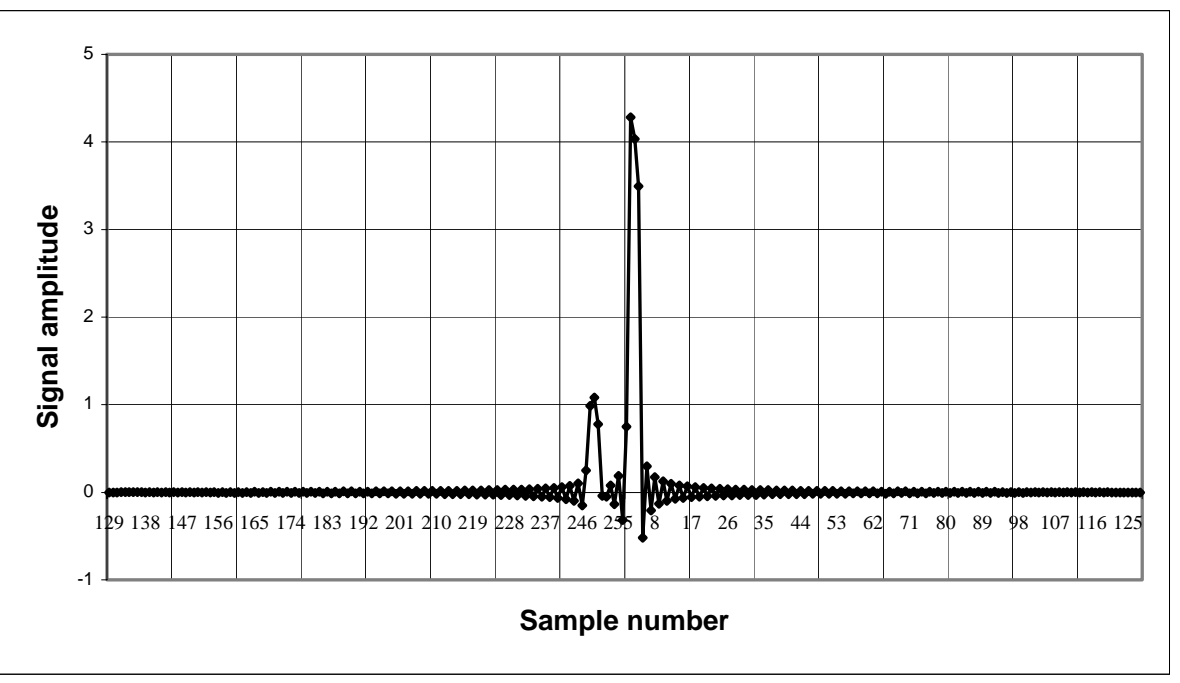

FIGURE 2. Signal reconstructed by the procedure (4a) or (4b), $\delta \approx 0.6515$.

Thus, in the cases in which measured bispectral data are available on a sparse set of points and a signal waveform is a priori unknown, conventional algorithm [1] may provoke large distortions in reconstructed signal shape.

Below we propose a new approach that permits to sufficiently decrease the distortions in the reconstructed signal waveform.

\section{PROPOSED APPROACH TO SIGNAL RECONSTRUCTION}

The main idea of our approach is to avoid the discontinuous phase function $\beta_{p, q}$ from the signal waveform recovery procedure. We propose: first, to replace the function $\beta_{p, q}$ with smooth and continuous functions as $\cos \beta_{p, q}$ and $\sin \beta_{p, q}$, and, second, to recover $\cos \hat{\varphi}_{p+q}$ and $\sin \hat{\varphi}_{p+q}$ instead of $\hat{\varphi}_{p+q}$ in the conventional procedure (3).

The proposed algorithm is based on rewriting the equation (1) using normalized bispectrum as 


$$
\begin{aligned}
\frac{B_{p, q}}{\left|B_{p, q}\right|}= & \cos \beta_{p, q}+j \sin \beta_{p, q}=\left(\cos \hat{\varphi}_{p}+j \sin \hat{\varphi}_{p}\right) \times \\
& \times\left(\cos \hat{\varphi}_{q}+j \sin \hat{\varphi}_{q}\right)\left(\cos \hat{\varphi}_{p+q}-j \sin \hat{\varphi}_{p+q}\right)
\end{aligned}
$$

From equation (6) the recovery procedures for cosine and sine of signal Fourier phase from cosine and sine of phase bispectrum can be written as

$$
\begin{aligned}
\cos \hat{\varphi}_{p+q}= & \left(\cos \hat{\varphi}_{p} \cos \hat{\varphi}_{q}-\sin \hat{\varphi}_{p} \sin \hat{\varphi}_{q}\right) \cos \beta_{p, q}+ \\
& +\left(\cos \hat{\varphi}_{p} \sin \hat{\varphi}_{q}+\sin \hat{\varphi}_{p} \cos \hat{\varphi}_{q}\right) \sin \beta_{p, q}, \\
\sin \hat{\varphi}_{p+q}= & \left(\cos \hat{\varphi}_{p} \sin \hat{\varphi}_{q}+\sin \hat{\varphi}_{p} \cos \hat{\varphi}_{q}\right) \cos \beta_{p, q}- \\
& -\left(\cos \hat{\varphi}_{p} \cos \hat{\varphi}_{q}-\sin \hat{\varphi}_{p} \sin \hat{\varphi}_{q}\right) \sin \beta_{p, q} .
\end{aligned}
$$

Just as in the conventional approach (see equations (4a) and (4b)), we propose below two different sequences of recursive steps. The first one, in accordance with (7a) and (7b) (parallel to the frequency axis $p=0$ ), can be defined as

$$
\begin{aligned}
& \left\{\begin{array}{l}
\cos \hat{\varphi}_{2}=\cos \beta_{1,1} \\
\sin \hat{\varphi}_{2}=-\sin \beta_{1,1}
\end{array}\right. \\
& \left\{\begin{array}{l}
\cos \hat{\varphi}_{N}=\cos \hat{\varphi}_{N-1} \cos \beta_{1, N-1}+\sin \hat{\varphi}_{N-1} \sin \beta_{1, N-1} \\
\sin \hat{\varphi}_{N}=\sin \hat{\varphi}_{N-1} \cos \beta_{1, N-1}-\cos \hat{\varphi}_{N-1} \sin \beta_{1, N-1}
\end{array} .\right. \\
& \left\{\begin{aligned}
\cos \hat{\varphi}_{N}= & \left(\cos \hat{\varphi}_{\frac{N-1}{2}} \cos \hat{\varphi}_{\frac{N+1}{2}}-\sin \hat{\varphi}_{\frac{N-1}{2}} \sin \hat{\varphi}_{\frac{N+1}{2}}\right) \cos \beta_{\frac{N-1}{2}, \frac{N+1}{2}}+ \\
& +\left(\sin \hat{\varphi}_{\frac{N-1}{2}} \cos \hat{\varphi}_{\frac{N+1}{2}}+\cos \hat{\varphi}_{\frac{N-1}{2}} \sin \hat{\varphi}_{\frac{N+1}{2}}\right) \sin \beta_{\frac{N-1}{2}, \frac{N+1}{2}} \\
\sin \hat{\varphi}_{N}= & \left(\sin \hat{\varphi}_{\frac{N-1}{2}} \cos \hat{\varphi}_{\frac{N+1}{2}}+\cos \hat{\varphi}_{\frac{N-1}{2}} \sin \hat{\varphi}_{\frac{N+1}{2}}\right) \cos \beta_{\frac{N-1}{2}, \frac{N+1}{2}}+ \\
& +\left(\sin \hat{\varphi}_{\frac{N-1}{2}} \sin \hat{\varphi}_{\frac{N+1}{2}}-\cos \hat{\varphi}_{\frac{N-1}{2}} \cos \hat{\varphi}_{\frac{N+1}{2}}\right) \sin \beta_{\frac{N-1}{2}, \frac{N+1}{2}}
\end{aligned}\right.
\end{aligned}
$$

Since the sequence of recursive steps in the direction parallel to the axis $q=0$ differs from (8) only by succession of the equations, we omit the equations corresponding to the sequence of recursive steps parallel to the frequency axis $q=0$. See the reference on how the samples are actually collected from the main bispectrum triangular domain in [1] where detailed illustrations of recursive strategy are given.

To compare the performance of the conventional algorithm employing the procedures (4a) and (4b) and the proposed algorithm using (8), we begin with example of single pulse signals with different pulse lengths $\Delta t$ and centered at the origin.

As a comparative example, the signal of the length of $\Delta t=37$ samples and amplitude of $A=1$ reconstructed by algorithm (8) is shown in Fig. 3. It is clearly seen from Fig. 3, that original rectangular signal waveform is not distorted. 
Note that both proposed procedures (parallel to the frequency axis $p=0$ or $q=0$ ) give errorless results for other considered pulse lengths $\Delta t$.

In Fig. 4 two-pulse signal (pulse lengths $\Delta t_{1}=\Delta t_{2}=3$ samples, pulse amplitudes $A_{1}=1$ and $A_{2}=4$ and the mutual center shift are the same as in Fig. 2) recovered by the proposed procedures is shown. Comparison of two-pulse signal waveform reconstructed by conventional procedure (4a) or (4b) (see Fig. 2) and by the abovementioned proposed procedures (see Fig. 4) permits to notice significant enhancement of the reconstructed signal quality due to application of the proposed algorithm.

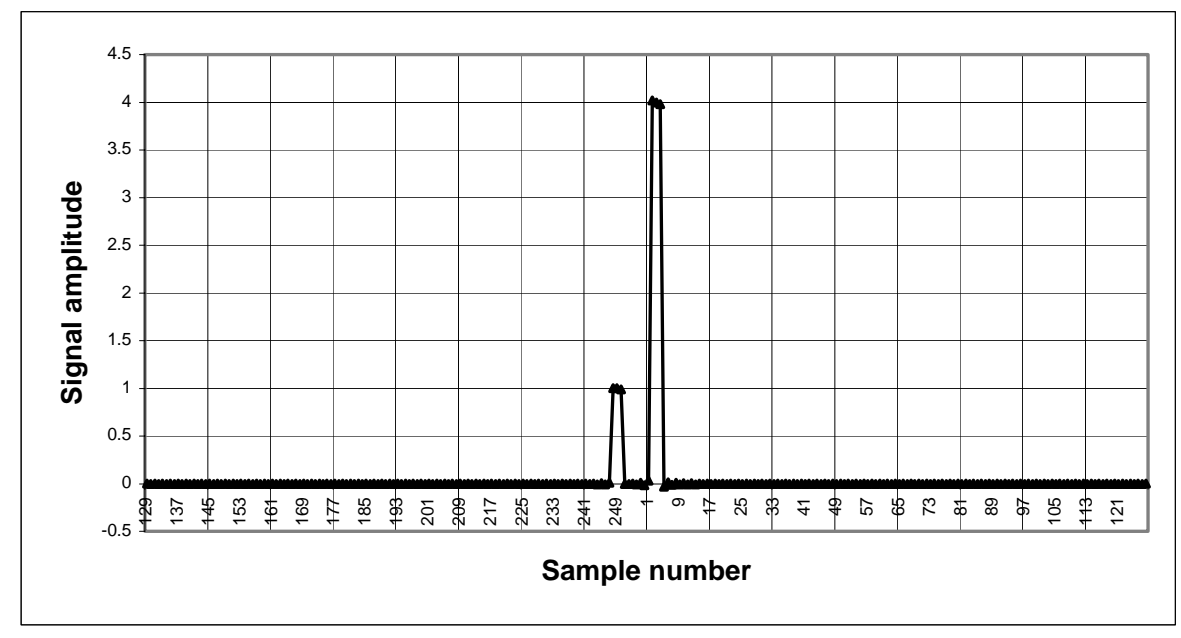

FIGURE 4. Signal reconstructed by the proposed procedure (8), $\delta=0.0335$.

\section{CONCLUSIONS}

A new simple approach to solving the bispectrum-based problem of reconstruction of an unknown signal waveform is considered and investigated. The main distinction of our approach from the known approaches is in abandonment of recovery of phase information, because the phase is not in itself interesting for signal reconstruction from bispectrum. In our opinion, traditional forming a bispectrum phase is unnecessary since we need only the normalized bispectrum for solving a reconstruction problem. The use of normalized bispectrum is free from typical constraints imposed on the processed signals and gives significant reduction of reconstructed signal distortions.

The proposed approach gives us a possibility to decrease algorithm sensitivity to sampling rate in bispectrum domain that is an important feature for reconstruction of an unknown signal waveform. The sampling frequency in our approach can be chosen approximately by Nyquist criterion and no extension of data file by zero padding is required for sampling errors decreasing. Hence, the proposed algorithm is computationally effective and one of its main advantages is simplicity. The results of numerical simulation indicate significant reduction of distortions in comparison to conventional technique. 


\section{REFERENCES}

1. Bartelt, H., Lohmann, A.W., and Wirnitzer B., (1984), Phase and Amplitude Recovery from Bispectra, Applied Optics, 23:3121-3129.

2. Sundaramoorthy, G., Raghuveer, M. R., and Dianat, S. A., (1990), Bispectral Reconstruction of Signals in Noise: Amplitude Reconstruction Issues, IEEE Trans. Acoustics, Speech, and Signal Processing, 38(7):1297-1306.

3. Kang, M.G., Lay, K.T., and Katsaggelos, A., (1991), Phase Estimation Using the Bispectrum and its Application to Image Restoration, Optical Engineering, 30(7):976-985.

4. Petropulu, A.P., and Nikias, C.L., (1992), Signal Reconstruction from the Phase of the Bispectrum, IEEE Transactions on Signal Processing, 40(3):601-610.

5. Alshebeili, S., and Cetin, A.E., (1990), A Phase Reconstruction Algorithm from Bispectrum, IEEE Transactions on Geoscience and Remote Sensing, 28(2):166-170.

6. Petropulu, A.P., and Pozidis, H., (1998), Phase Reconstruction from bispectrum slices, IEEE Transactions on Signal Processing, 46(2):527-530.

7. Nikias, C.L. and Raghuveer, M.R., (1987), Bispectral estimation: A digital signal processing framework, Proc. IEEE, 75(7):869-891. 